\title{
Determinación Computacional del Coeficiente de Transferencia de Calor en Calentadores Eléctricos de Flujo Continuo, mediante Dinámica de Fluidos Computacional
}

\author{
Valaur E. Márquez-Baños ${ }^{(1)}$, José. J. Valencia-López ${ }^{(2)}$, Oscar García-Aranda( ${ }^{(1)}$ y Christopher Heard ${ }^{(3)}$ \\ (1) Posgrado en Ciencias Naturales e Ingeniería, Univ. Autónoma Metropolitana Cuajimalpa, México. \\ (2) Departamento de Procesos y Tecnología, Univ. Autónoma Metropolitana Cuajimalpa, México. \\ (e-mail: jvalencia@correo.cua.uam.mx) \\ (3) Departamento de Teoría y Procesos de Diseño, Univ. Autónoma Metropolitana Cuajimalpa., Av. Vasco \\ de Quiroga 4871, Cuajimalpa de Morelos, Santa Fe Cuajimalpa, 05348 Ciudad de México, México.
}

Recibido Dic. 10, 2015; Aceptado Ene. 27, 2016; Versión final Mar. 28, 2016, Publicado Oct. 2016

\section{Resumen}

Se realizó la estimación del coeficiente de transferencia de calor mediante herramientas de Dinámica de Fluidos Computacional (CFD) para cuatro configuraciones de calentadores eléctricos de 5,08 cm de diámetro: a) tubo simple, b) bafles verticales, c) bafle helicoidal, y d) bafle helicoidal, con variación de torsión. El modelo computacional se planteó empleando el modelo $k-\varepsilon$ para representar los fenómenos de turbulencia acoplado con la ecuación del balance de calor. Los coeficientes de transferencia de calor obtenidos mostraron similitud entre las configuraciones a, c y d. La configuración b presentó un aumento significativo debido a la turbulencia generada dentro de la sección interna en los bafles, y mostró también una elevada caída de presión.

Palabras clave: calentador eléctrico; coeficiente de transferencia de calor; dinámica de fluidos computacional; elemento finito

\section{Computational Determination of Heat Transfer Coefficient in Continuous Flow Electrical Heaters, using Computational Fluid Dynamics}

\begin{abstract}
The estimation of the heat transfer coefficient using tools of Computational Fluid Dynamics (CFD) for four configurations of electric heaters of $5.08 \mathrm{~cm}$ in diameter has been done: a) single pipe, b) vertical baffles, c) helical baffle, d) helical baffle with torsion variation. The computational model is established using the $\mathrm{K}-\varepsilon$ model to represent the phenomena of turbulence coupled with the heat balance equation. The heat transfer coefficients obtained by this method showed similarity between configurations $a, c$ and $d$. The configuration $b$ presented a significant increase due to the turbulence generated within the internal section in the enclosures, and showed a high pressure drop.
\end{abstract}

Keywords: electric heaters; heat transfer coefficient; computational fluid dynamics; finite element 


\section{INTRODUCCIÓN}

La optimización de los procesos de transferencia de calor reviste una gran importancia para el ahorro de energía y la reducción de la contaminación (Omer, 2008; Friedler, 2010). Dentro de los equipos de transferencia de calor de uso común se encuentran los calentadores eléctricos, los cuales pueden ser de uso doméstico, siendo usados para elevar la temperatura del agua a condiciones más cómodas para su uso (Reichl y Pleschinger, 2013), o evitar su congelamiento en tuberías durante el invierno (Jīn, 2005). El uso industrial de este tipo de calentadores es variado, desde el calentamiento de gases y solventes, hasta el calentamiento de corrientes de líquidos de proceso (Su, 2011).

Tan solo en Estados Unidos (EU), los calentadores eléctricos de agua ocupan el segundo lugar en consumo de energía en los hogares y representan el $17 \%$ del consumo de energía residencial, llegando significar hasta $\$ 200$ a $\$ 600$ al año por casa (EIA, 2015). En 2009 fueron vendidos un total de 8 millones de calentadores de agua para hogares tan solo en EU, siendo los calentadores de agua con almacenamiento el tipo más común a la venta, dominando el $96 \%$ del mercado. Los calentadores de agua continuos, que sirven para toda el agua en el hogar o para un lugar en específico, representan el $4 \%$ restante de las ventas, pero están aumentando de popularidad (Ryan et al., 2010). A pesar de esta importancia comercial, los calentadores eléctricos han sido poco estudiados para una mejor comprensión y su optimización, siendo prácticamente nula la cantidad de trabajos académicos que prestan atención a este tipo de sistemas.

Un método desarrollado para la mejora de los procesos de transferencia de calor es la inclusión de bafles en la zona de intercambio y más recientemente el uso de bafles helicoidales (Taher et al., 2012; RodríguezToral y Heard, 2012; Wen et al., 2015). El uso bafles helicoidales fue desarrollado por primera vez en Checoslovaquia para intercambiadores de calor de tubos y coraza. Estos intercambiadores de calor, llamados también "helixchangers" (Nasr y Shafeghat, 2008), han sido capaces de minimizar los principales defectos de intercambiadores de calor de coraza y tubos con deflectores verticales y han mostrado un rendimiento muy eficaz sobre todo para los casos en los que se controla el coeficiente de transferencia de calor en el lado de la coraza; teniendo una menor caída de presión y ensuciamiento (Lei et al., 2008; Movassag et al., 2013). También han mostrado ser eficaces bajo condiciones de vibración (Weaver y Fitzpatrick, 1988; Goyder, 2002). En la literatura científica aparecen numerosos trabajos donde se evalúan diversas mejoras a estos equipos. La mayoría de estos trabajos se enfoca la optimización hacia un solo objetivo, por lo general el aumento del coeficiente de transferencia de calor (Dong et al., 2014; Wang et al., 2014; Dong et al., 2015) o la disminución de la potencia de bombeo (Lei et al., 2008; Ozden y Tari, 2010) mediante cambios en la configuración interna del equipo, por lo que la inclusión de bafles en calentadores eléctricos de flujo continuos es una alternativa para aumentar la eficacia de estos calentadores, buscando mejoras en el coeficiente de transferencia de calor.

El cálculo de coeficiente de calor generalmente requiere de experimentación y un posterior análisis de los datos para obtener dicho valor (Alvis et al., 2010). Sin embargo, una estrategia para evaluar este tipo de sistemas consiste en la simulación basada en herramientas de Dinámica de Fluidos Computacional (CFD, por sus siglas en inglés), la cual puede proporcionar información del campo de flujo y la distribución de temperaturas a un costo mucho más bajo en comparación con una investigación experimental integral, debido al alto coste del equipo.

El avance en los equipos de cómputo actuales hace que sea posible la simulación numérica de un intercambiador de calor en su totalidad (Bhutta et al., 2012). Apoyándose en ello, diversos autores han realizado estimaciones del coeficiente de transferencia de calor en intercambiadores con bafles verticales y sistemas con bafles helicoidales a través de simulaciones (Zhang, et al., 2009; You et al., 2012; Xiao et al., 2013; Chen et al., 2013; Yang, et al., 2014). No obstante, el cálculo del coeficiente de transferencia de calor en calentadores eléctricos aún no ha sido abordado, por ello el presente trabajo tiene como objetivo calcular el coeficiente de transferencia de calor en calentadores eléctricos de flujo continuo mediante herramientas de Dinámica de Fluidos Computacional, con la finalidad de establecer una relación entre los aspectos geométricos (presencia de bafles) y de operación (flujo) sobre la transferencia de calor e hidrodinámica de los sistemas.

\section{MATERIALES Y MÉTODOS}

En el presente trabajo se estudiaron cuatro configuraciones de calentadores eléctricos. Los sistemas analizados cuentan con una resistencia interna como medio de calentamiento, en la Figura 1 se muestran las configuraciones propuestas en el presente trabajo, a) tubo simple, b) bafles verticales, c) bafle helicoidal, d) bafle helicoidal con variación de torsión. Cada uno presenta una hidrodinámica particular, promoviendo una transferencia de calor y requerimientos de bombeo distintos. 


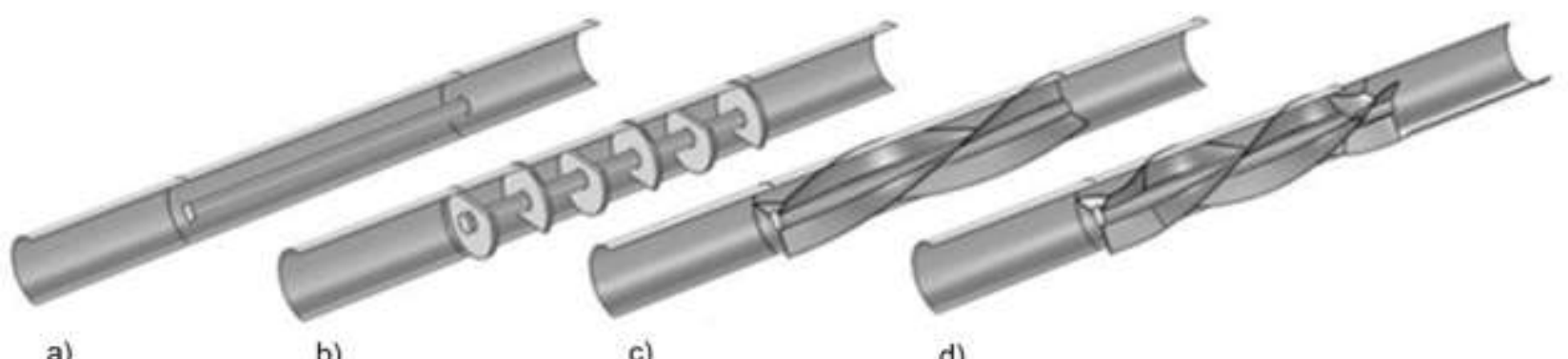

a)

b)

c)

d)

Fig. 1: Configuraciones de calentadores eléctricos: a) tubo simple, b) bafles verticales, c) bafle helicoidal, d) bafle helicoidal con variación de torsión.

Estos calentadores eléctricos poseen $5,08 \mathrm{~cm}$ de diámetro. La configuración a) es únicamente el ducto con la resistencia, la configuración b) posee 6 bafles verticales a lo largo de la resistencia, el sistema c) tiene como bafle una cinta helicoidal de 3 vías con torsión constante a un giro completo a lo largo de la resistencia y finalmente el sistema d) tiene un bafle helicoidal con variación de torsión, con lo cual se pretende reducir los efectos no deseados de vorticidad excesiva a la salida del intercambiador. El área de intercambio que posee cada sistema se presenta en la Tabla 1.

Tabla 1: Área de intercambio de cada configuración de calentador

\begin{tabular}{|l|c|}
\hline Geometría del calentador & Área de intercambio $\left(\mathrm{m}^{2}\right)$ \\
\hline a) tubo simple & 0,01090 \\
\hline b) bafles verticales & 0,03193 \\
\hline c) bafle helicoidal & 0,03837 \\
\hline d) bafle helicoidal con variación de torsión. & 0,03901 \\
\hline
\end{tabular}

\section{El modelo}

El desarrollo de este modelo computacional para el cálculo de coeficientes de transferencia de calor, se basó en los siguientes supuestos: (1) El fluido de trabajo es continuo, incompresible, isotrópico y newtoniano; (2) El efecto de la gravedad es despreciable; (3) Se ignora el calentamiento viscoso o radiación térmica; (4) El flujo está completamente desarrollado y ha alcanzado un estado estacionario; (5) Los calentadores eléctricos se encuentran operando en régimen turbulento a flujos de 3,78, 11,36, 26,5 y $53 \mathrm{l} / \mathrm{min}$.; (6) Las paredes del calentador se encuentran perfectamente aisladas; (7) La potencia de la resistencia es constante; y (8) No se presenta cambio de fase en el fluido de trabajo.

Para representar el movimiento del fluido en régimen turbulento, se aplicó el modelo $\mathrm{K}-\varepsilon$ estándar (Launder y Spalding, 1974; Andersson et al., 2012), descrito en las Ecuaciones (1) a (6).

$$
\frac{\partial \mathbf{u}}{\partial t}+\rho(\mathbf{u} \cdot \nabla) \mathbf{u}=\nabla \cdot\left[-p \mathbf{l}+\left(\mu+\mu_{T}\right)\left(\nabla \mathbf{u}+(\nabla \mathbf{u})^{T}\right)-\frac{2}{3} \rho \kappa \mathbf{l}\right]
$$

$\nabla \cdot(\mathbf{u})=0$

$\rho \frac{\partial \kappa}{\partial t}+\rho(\mathbf{u} \cdot \nabla) \kappa=\nabla \cdot\left[\left(\mu+\frac{\mu_{T}}{\sigma_{\kappa}}\right) \nabla \kappa\right]+P_{\kappa}-\rho \varepsilon$

$\rho \frac{\partial \varepsilon}{\partial t}+\rho(\mathbf{u} \cdot \nabla) \varepsilon=\nabla \cdot\left[\left(\mu+\frac{\mu_{T}}{\sigma_{\varepsilon}}\right) \nabla \varepsilon\right]+C_{\varepsilon 1} \frac{\varepsilon}{\kappa} P_{\kappa}-C_{\varepsilon 2} \rho \frac{\varepsilon^{2}}{\kappa}$

$\mu_{T}=\rho C_{\mu} \frac{\kappa^{2}}{\varepsilon}$

$P_{\kappa}=\mu_{T}\left[\nabla \mathbf{u}:\left(\nabla \mathbf{u}+(\nabla \mathbf{u})^{T}\right)\right]-\frac{2}{3} \rho \kappa \nabla \cdot \mathbf{u}$ 
Donde: $\mathbf{u}$ es el vector velocidad, $\rho$ es la densidad, $t$ es el tiempo, $\mu$ es la viscosidad del fluido, $\mu_{T}$ es la viscosidad turbulenta, $k$ es la energía cinética de turbulencia, $\varepsilon$ es disipación turbulenta, y $\sigma_{\varepsilon}, \sigma_{k}, C_{\varepsilon 1}, C_{\varepsilon 2}, C_{\mu}$, son constantes del modelo $\mathrm{K}-\varepsilon$.

Con condiciones de frontera:

En la entrada:

$\mathbf{u}=-U_{0} \mathbf{n}$

$U_{\text {ref }}=U_{0}$

$\kappa=\frac{2}{3}\left(U_{r e t} / T_{T}\right)^{2}, \varepsilon=C_{\mu}^{3 / 4} \frac{\kappa^{3 / 2}}{L_{T}}$

Donde: $U_{0}$ es la velocidad de entrada y $L_{T}$ e $I_{T}$ son la escala de longitud y la intensidad de turbulencia.

En la salida:

$\left[-p \mathbf{l}+\left(\mu+\mu_{T}\right)\left(\nabla \mathbf{u}+(\nabla \mathbf{u})^{T}\right)-\frac{2}{3} \rho \kappa \mathbf{l}\right] \mathbf{n}=-p_{0} \mathbf{n}$

$\nabla \kappa \cdot \mathbf{n}=0, \nabla \varepsilon \cdot \mathbf{n}=0$

Donde: $p_{0}$ es la presión de salida

En las paredes:

$\mathbf{u} \cdot \mathbf{n}=0$

$\nabla \kappa \cdot \mathbf{n}=0, \varepsilon=\rho \frac{C_{\mu} \kappa^{2}}{k_{v} \delta_{w}^{+} \mu}$

Donde: $k v$ es la constante de von Kárman y $\delta^{+}{ }_{w}$ es la distancia de pared.

El modelo $\kappa-\varepsilon$ estándar aplicado en este trabajo, es ampliamente utilizado en aplicaciones industriales y problemas de transferencia de calor debido a su economía y exactitud, requiriendo menor memoria y tiempo de cómputo durante las simulaciones en comparación con otros modelos descritos en la literatura (Andersson et al., 2012). Este modelo ha sido empleado con éxito en equipos de transferencia de calor para describir flujos mal distribuidos (Zhang y Li, 2003; Wen y Li, 2004; Wasewar et al., 2007; Kim et al., 2009), así como para predecir caídas de presión y estudiar coeficientes térmicos (Kumar et al., 2006; Kim et al., 2008; Ismail y Velraj, 2009; Lisboa et al., 2010; Shi et al., 2010; Yang et al., 2014;).

El balance de calor (Bird et al., 2006) está dado por la Ecuación (10):

$\rho C_{p} \frac{\partial T}{\partial t}+\rho C_{p} \mathbf{u} \cdot \nabla T=\nabla \cdot\left(\left(k+k_{T}\right) \cdot \nabla T\right)+Q$

Donde: $T$ es la temperatura, $k$ es la conductividad térmica, $C p$ es la capacidad calorífica, $Q$ es el término fuente de calor y $k_{T}$ es la conductividad de turbulencia, la cual se calcula mediante la correlación de KaysCrawford (Kays, 1994) para el número de Prandtl turbulento $(\operatorname{Pr} T)$ :

$\operatorname{Pr}_{T}=\frac{C p \mu_{T}}{k_{T}}=\left[\frac{1}{2 \operatorname{Pr}_{T \infty}}+\frac{0.3}{\sqrt{\operatorname{Pr}_{T \infty}}} \frac{C p \mu_{T}}{k}-\left(0.3 \frac{C p \mu_{T}}{k}\right)^{2}\left(1-\exp \left(\frac{-k}{\left(0.3 C p \mu_{T} \sqrt{\operatorname{Pr}_{T \infty}}\right)}\right)\right)\right]^{-1}$

Donde: $\operatorname{Pr}_{T_{\infty}}$, es el número de Prandtl turbulento en el infinito, con un valor de 0.85 .

Las condiciones de frontera para Ecuación (10) son:

En la entrada:

$T=T_{0}$ 
Donde: $\mathrm{T}_{0}$ es la temperatura de entrada del fluido, $20^{\circ} \mathrm{C}$.

En la salida:

$-\mathbf{n} \cdot(-k \nabla T)=0$

En las paredes:

$-\mathbf{n} \cdot(-k \nabla T)=0$

Como fuente de calor se implantó $Q=24 \mathrm{~kW}$ sobre la zona de la resistencia central, valor de potencia común en los calentadores comerciales que operan a tasas de flujo como las analizadas en este trabajo (Elecro, 2016). Como fluido de análisis se ha tomado al agua y los bafles del intercambiador se consideran de aluminio. Las propiedades de ambos con dependencia de la temperatura son tomadas de la base de datos de COMSOL (COMSOL Inc., 2016).

El coeficiente transferencia de calor $(h)$ es calculado como:

$$
h=\frac{Q}{A\left(T_{w}-T_{\infty}\right)}
$$

Donde: $Q$ es el calor subministrado al fluido, $A$ el área de intercambio, $T_{w}$ la temperatura de pared de la resistencia y los bafles, $T_{\infty}$ es temperatura promedio en el seno del fluido.

\section{Solución}

Estas ecuaciones son resueltas de manera acoplada en toda la geometría de los intercambiadores, empleando el método de elementos finitos disponible en COMSOL Multiphysics 5.1. Para cada sistema se construyeron mallas formadas de elementos tetraédricos. El número de elementos en las mallas para cada geometría analizada se presenta en la Tabla 2. La independencia de la malla fue obtenida analizando el caso con el flujo de $53 \mathrm{l} / \mathrm{min}$, hasta obtener cambios menores al 1,5\% en los valores de la caída de presión y flujo de calor, respecto a una malla con menor número de elementos.

Tabla 2: Tamaños de malla usados en las simulaciones de cada calentador de calor.

\begin{tabular}{|l|c|}
\hline Geometría del calentador & Número de elementos \\
\hline a) tubo simple & 92325 \\
\hline b) bafles verticales & 101276 \\
\hline c) bafle helicoidal & 160671 \\
\hline d) bafle helicoidal con variación de torsión. & 235294 \\
\hline
\end{tabular}

En las ecuaciones de movimiento fue empleada una discretización tipo $\mathrm{P} 1+\mathrm{P} 1$ y de segundo orden para la temperatura, como método de solución fue empleado un resolvedor directo tipo PARDISO (PARallel Dlrect SOlver), para la solución del sistema en estado estacionario de modo segregado con una tolerancia relativa de 0.001 (Petra et al., 2014).

\section{RESULTADOS Y DISCUSIÓN}

Los resultados se muestran en forma separada pàra los Perfiles de temperatura, la Hidrodinámica, las Caídas de presión y los Coeficientes de transferencia de calor

\section{Perfiles de temperatura}

En la Figura 2 se presentan las temperaturas de salida de cada uno de los calentadores analizados, en esta imagen se puede apreciar que la temperatura alcanzada en los diferentes calentadores en estado estacionario es similar, siendo ligeramente menor en el calentador de tubo simple. En cambio en la Figura 3, los perfiles de temperatura en estado estacionario para las configuraciones analizadas para un flujo de operación de $53 \mathrm{l} / \mathrm{min}$, muestran que las temperaturas alcanzadas en la pared de la zona de calentamiento son distintas en cada configuración, siendo evidente un elevado calentamiento de la resistencia, cuando no presenta bafles que ayuden a la distribución de calor. 


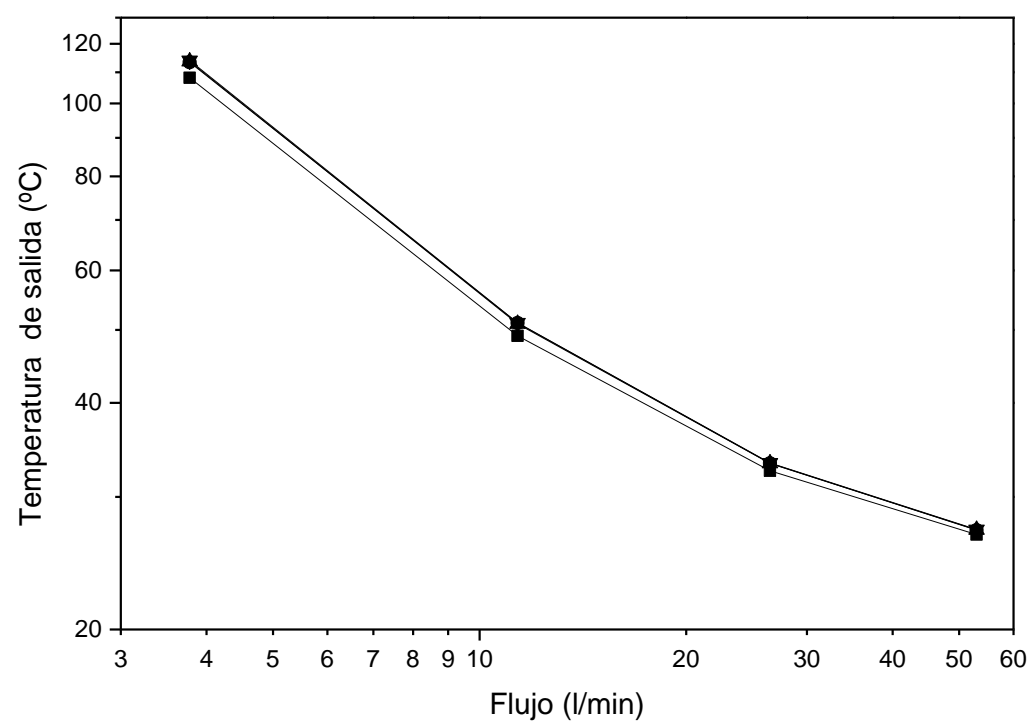

Fig. 2: Temperatura a la salida de los calentadores: tubo simple ( $\bullet)$, bafles verticales $(\bullet)$, bafle helicoidal $(\boldsymbol{\Lambda})$, bafle helicoidal con variación de torsión $(\mathbf{v})$.
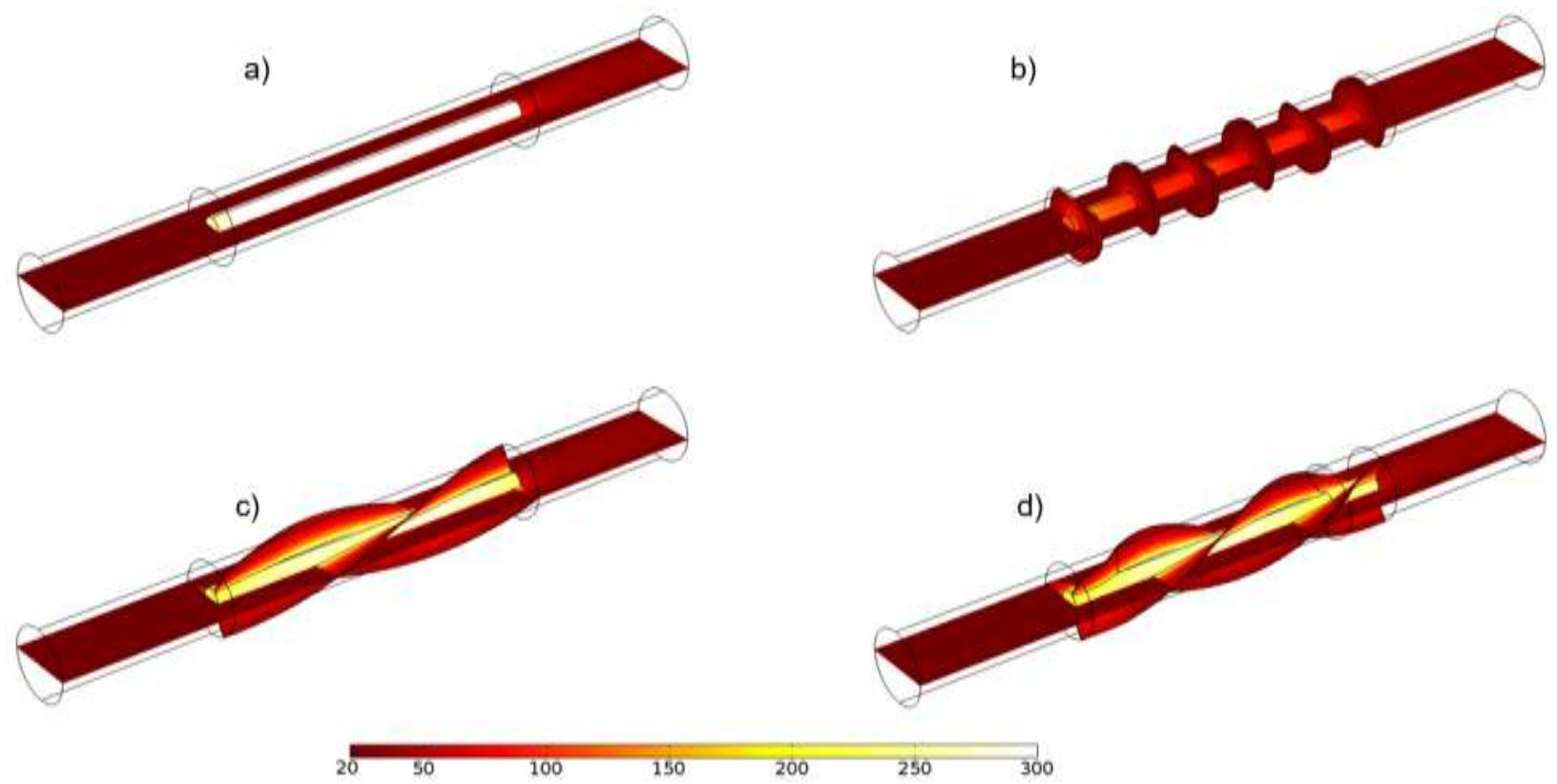

Fig. 3: Perfiles de temperatura $\left({ }^{\circ} \mathrm{C}\right)$ para los calentadores analizados: a) tubo simple, b) bafles verticales, c) bafle helicoidal, d) bafle helicoidal con variación de torsión.

\section{Hidrodinámica}

En la Figura 4 se muestran los patrones de flujo para los sistemas analizados para un flujo de operación de 53 $\mathrm{l} / \mathrm{min}$. En esta figura se puede observar que para el caso del calentador de tubo simple, el fluido se acelera ligeramente en la sección anular entre la resistencia y la pared del tubo, permaneciendo un flujo en dirección normal a la entrada. Para el caso de los calentadores dotados con bafles helicoidales, es posible notar que el fluido presenta una mayor aceleración, sin que existan cambios bruscos de velocidad, reduciendo la posibilidad de formación de remolinos y turbulencia (Movassag et al., 2013; Dong et al., 2015). Para el caso del calentador con el bafle helicoidal con variación de torsión, el flujo es presenta una velocidad más homogénea en comparación al resto de los calentadores analizados. Por otro lado, en el del calentador con bafles verticales el flujo presenta grandes recirculaciones en cada sección de los bafles, con cambios de velocidad bruscos que ocasionan aceleraciones y desaceleraciones de velocidad de flujo en cada cambio de sección, creando una turbulencia elevada en estas regiones, tal como lo muestra la Figura $5 \mathrm{~b}$.

La presencia de estos remolinos y la elevada turbulencia en calentador con bafles verticales, puede ocasionar inestabilidad de flujo, provocando problemas de vibración (Goyder, 2002). Este problema se ve disminuido 
mediante el uso de los bafles helicoidales, al presentarse una energía cinética de turbulencia 3 órdenes de magnitud menor en estos sistemas y un flujo más ordenado, como se distingue en el resto de las imágenes de la Figura 5.
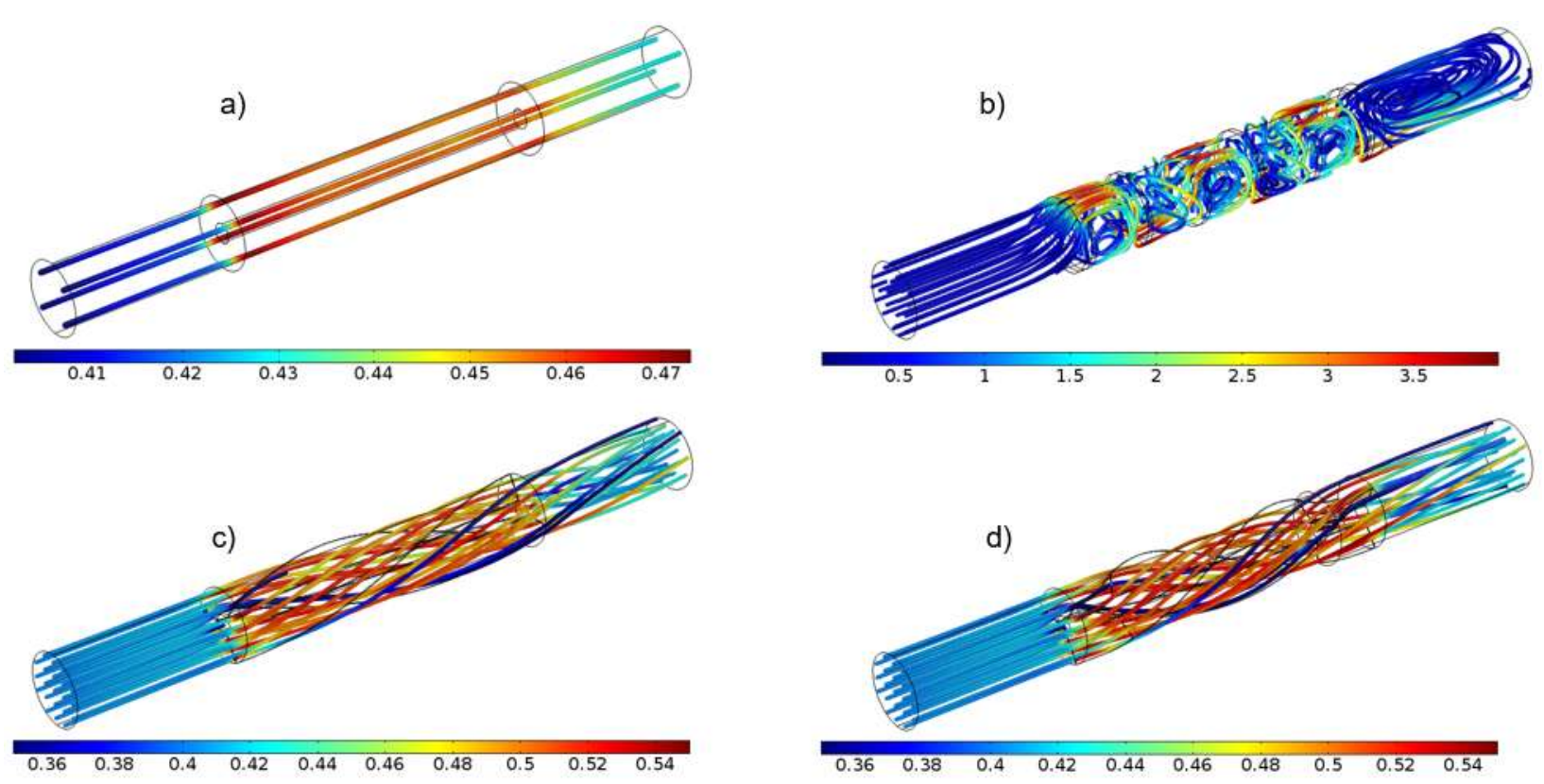

Fig. 4: Líneas de corriente con tono de velocidad (m/s) para los calentadores analizados: a) tubo simple, b) bafles verticales, c) bafle helicoidal, d) bafle helicoidal con variación de torsión.
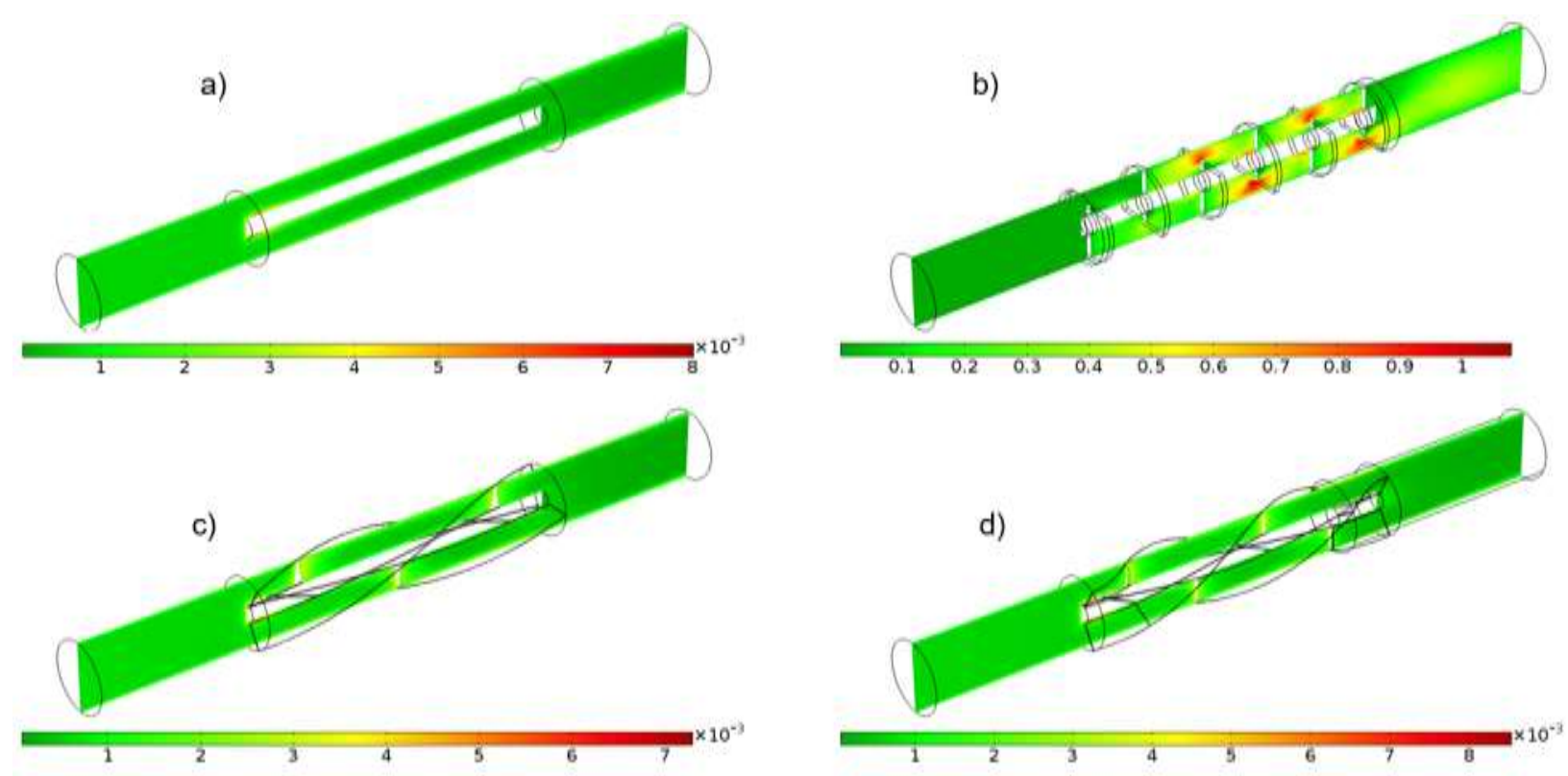

Fig. 5: Perfiles de energía cinética de turbulencia $\left(\mathrm{m}^{2} / \mathrm{s}^{2}\right)$ para los calentadores analizados: a) tubo simple, b) bafles verticales, c) bafle helicoidal, d) bafle helicoidal con variación de torsión.

Las líneas de corriente del calentador con bafles helicoidales de la Figura 4c muestran giros y baja uniformidad en la velocidad a la salida del calentador, lo cual es reducido con el uso de una hélice con torsión variable (Fig. 4d). Esto se distingue de una mejor manera en la Figura 6, en forma de perfiles de vorticidad, indicándonos que en la configuración con bafles helicoidales con variación de torsión, el flujo tiende a ser normal al área transversal del ducto, lo cual genera un perfil de velocidad adecuado para la conexión a otros equipos, evitando así recirculación cerca de la alimentación. 


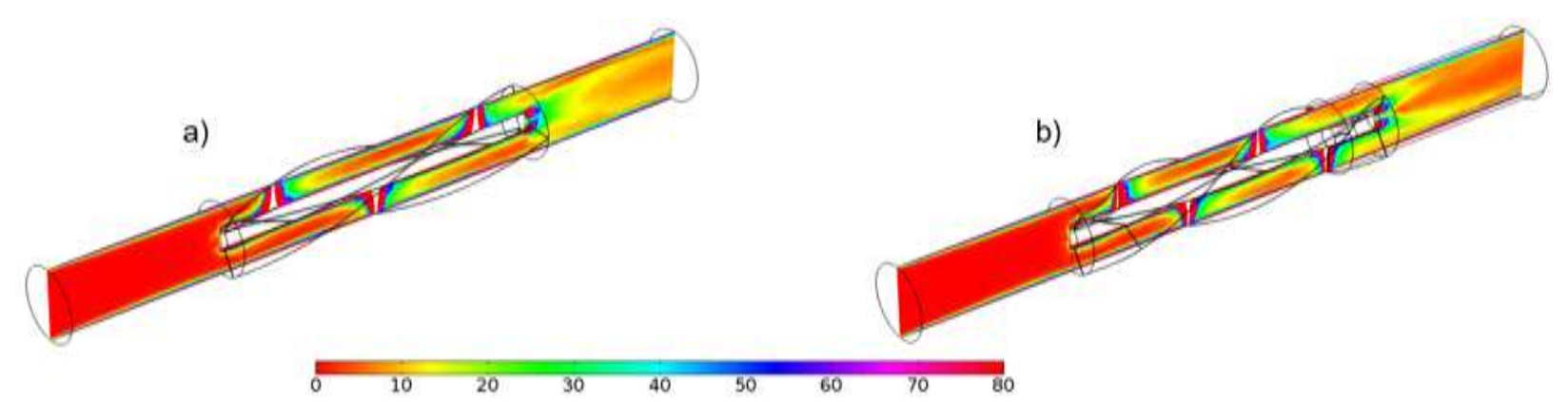

Fig. 6: Perfiles de vorticidad $\left(\mathrm{s}^{-1}\right)$ para los calentadores analizados: a) bafle helicoidal, b) bafle helicoidal con variación de torsión.

\section{Caídas de presión}

La caída de presión es de gran importancia en el diseño de los equipos de trasferencia de calor, porque los costes de bombeo son altamente dependientes de la caída de presión (Taher et al., 2012) y por lo tanto, una menor caída de presión podría los reducir costos de operación. En la Figura 7 se muestra la caída de presión que presenta cada sistema al incrementar el flujo.

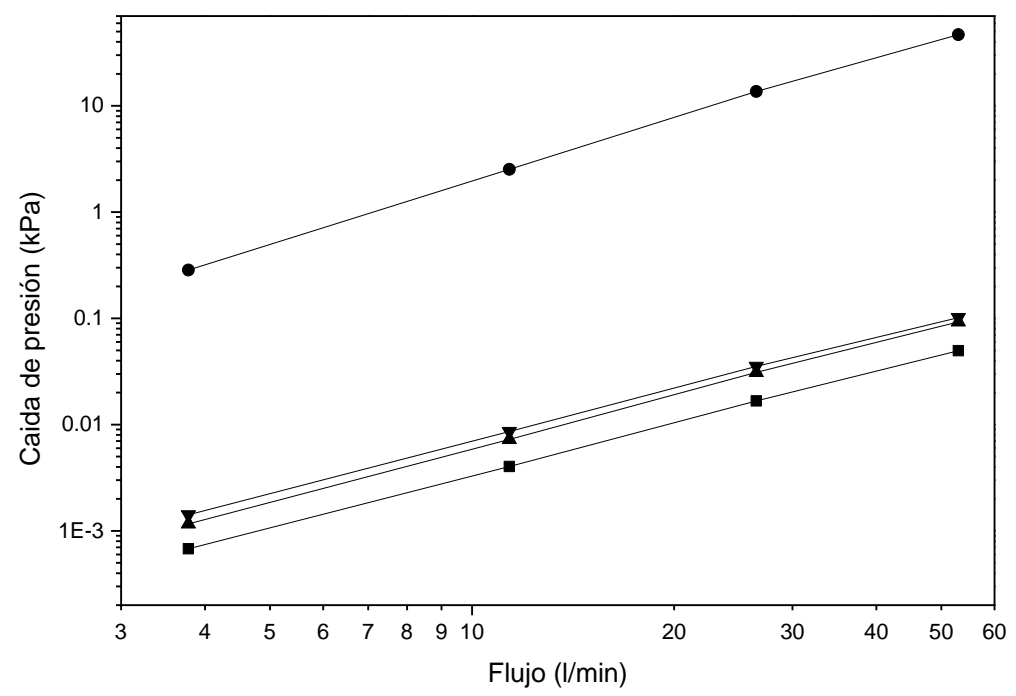

Fig. 7: Caída de presión (kPa) para los calentadores analizados: tubo simple (₫), bafles verticales $(\bullet)$, bafle helicoidal ( $\mathbf{\Lambda})$, bafle helicoidal con variación de torsión $(\boldsymbol{\nabla})$.

En ella se puede apreciar que en el calentador de bafles verticales, se obtiene una caída de presión excesiva, siendo de uno a dos órdenes de magnitud mayor en comparación con los otros calentadores. Una de las principales causas de las altas caídas de presión con bafles verticales, son los cambios bruscos de velocidad entre el flujo en la ventana (donde se da un giro de $180^{\circ}$ ) y la cámara entre bafles, generando recirculaciones y turbulencia. Dicho flujo turbulento y con remolinos es una fuente importante de caída de presión y requiere de energía de bombeo (Rodríguez-Toral y Heard, 2012). En la Figura 8b se presenta el perfil de presión para este calentador, en donde se distingue que en cada paso por los bafles se genera una caída de presión significativa.

Por otro lado, el uso de los bafles helicoidales genera una caída de presión menor, debido a la aceleración uniforme del fluido sobre las vías de los bafles, produciendo un incremento gradual en la caída de presión, tal como se distingue en la Figura 8. En cuanto al calentador de tubo simple, su caída de presión es la menor de todos los sistemas debido la ausencia de aditamentos internos que incrementen la necesidad de bombeo.

Los resultados obtenidos para la caída de presión del calentador de bafles verticales, muestran concordancia con los valores reportados experimentalmente por diversos autores para sistemas similares (Zhang et al., 2009; Wang et al., 2011; Xiao et al., 2013; Zhang et al., 2013). La mayoría de ellos en un intervalo de 2 a $60 \mathrm{kPa}$, a un régimen de flujo como el del presente trabajo. En cuanto a las caídas de presión de los calentadores con bafles helicoidales, no existen datos para disponibles para sistemas de 3 vías. Sin embargo, los valores obtenidos son de una magnitud comprable con los reportados por Yang et al. (2014), para un intercambiador con un bafle helicoidal de un solo giro. 

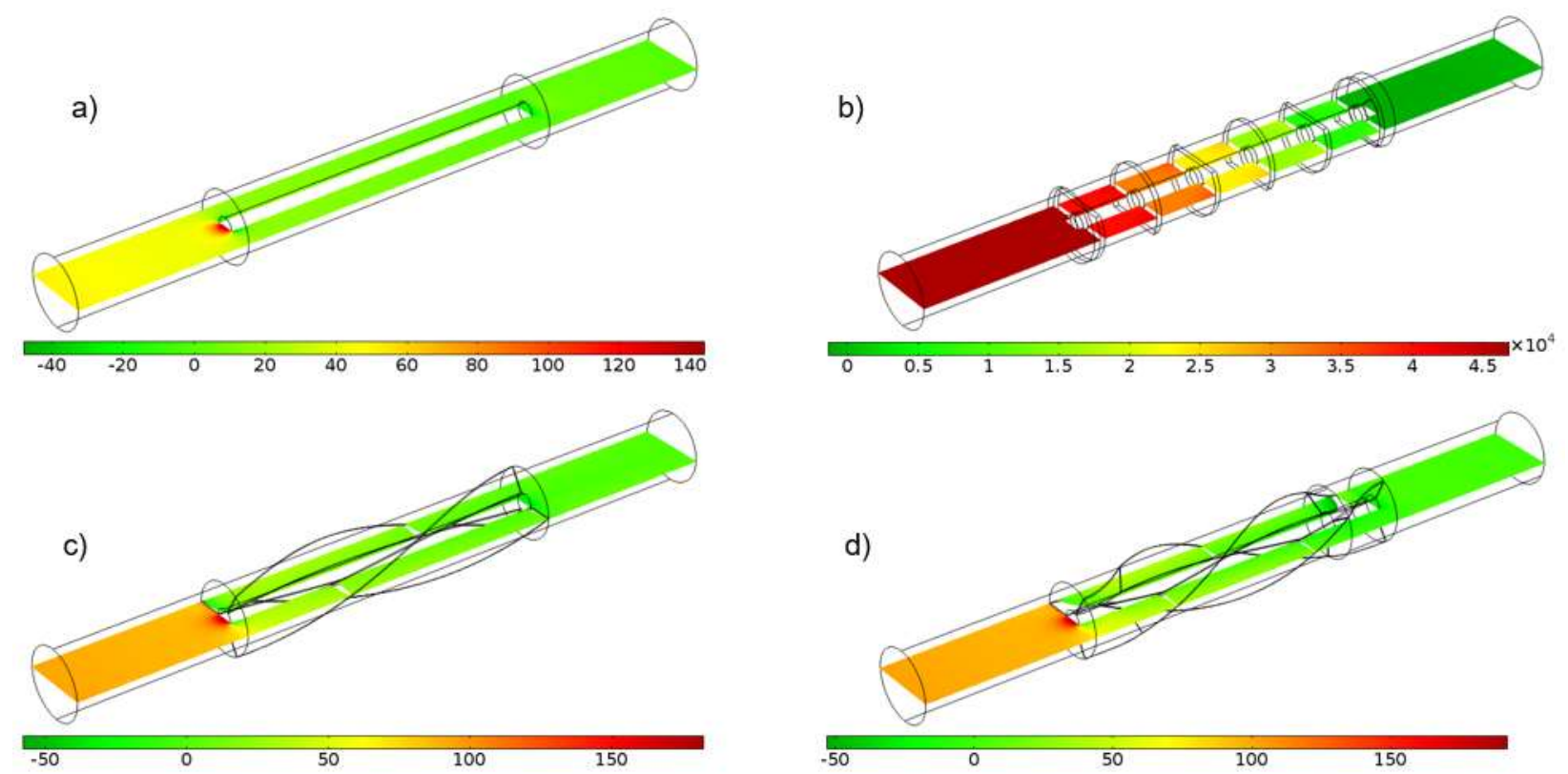

Fig. 8: Perfiles de presión (Pa) para los calentadores analizados: a) tubo simple, b) bafles verticales, c) bafle helicoidal, d) bafle helicoidal con variación de torsión.

\section{Coeficientes de transferencia de calor}

En la Figura 6 se muestran los valores calculados para el coeficiente de transferencia de calor $(h)$ a las condiciones de operación analizadas. En ella se puede notar que el valor de $h$ es muy simular para las configuraciones de bafle helicoidal y tubo simple, notándose una ventaja sobre estos en el caso del bafle con variación de torsión. No obstante, para el calentador con bafles verticales el coeficiente es casi 10 veces mayor. Esto puede ser atribuido a la alta turbulencia generada en cada una de las secciones entre los bafles, tal como se mostró en la Figura 5 como perfiles de energía cinética de turbulencia.

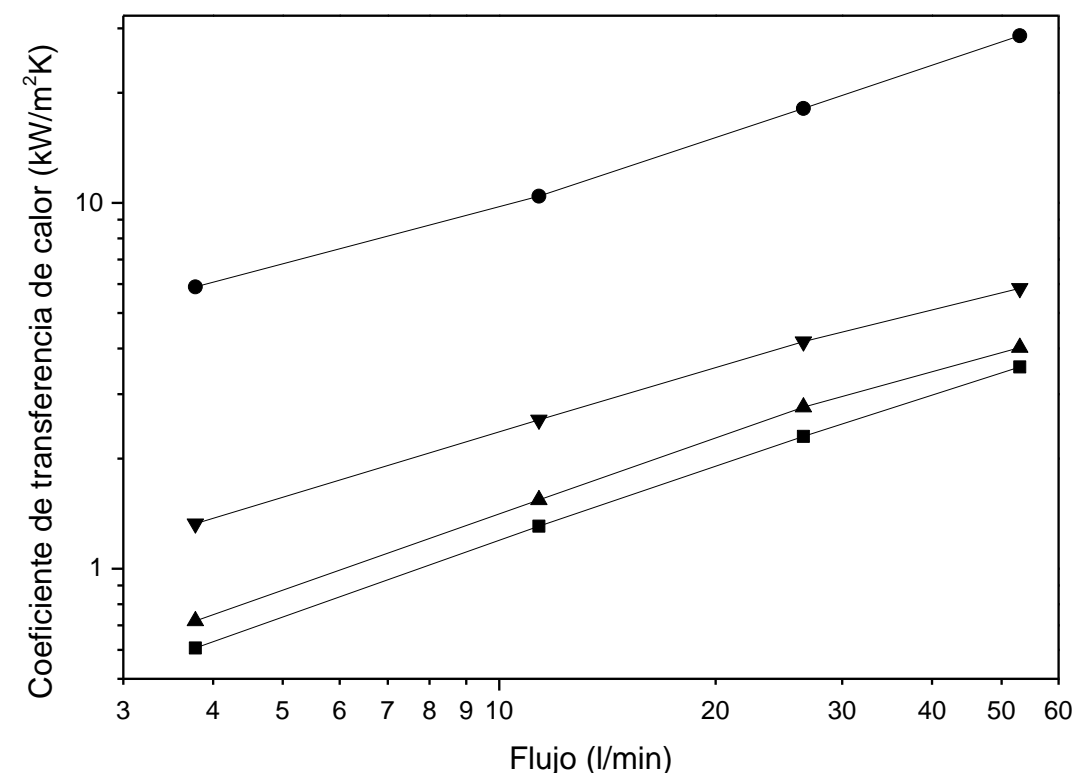

Fig. 9: Coeficiente de transferencia de calor en función del flujo volumétrico: tubo simple (๑), bafles verticales (•), bafle helicoidal $(\mathbf{\Lambda})$, bafle helicoidal con variación de torsión $(\mathbf{\nabla})$.

La exagerada caída de presión que presenta el sistema con bafles helicoidales, ocasiona que este sistema no sean tan atractivo a pesar de poseer un coeficiente de transferencia de calor mucho mayor, en comparación a los otros calentadores, basándonos en este criterio el calentador con el bafle helicoidal con variación de torsión es la mejor configuración de las analizadas en el presente trabajo. 
En la literatura ha sido reportado por varios autores que los sistemas con bafles helicoidales tienden a poseer coeficientes de transferencia de calor menores a los de bafles verticales (Zhang et al., 2009; Movassag et al., 2013; Zhang et al., 2013), con la ventaja de que su caída presión es menor y la transferencia de calor puede ser controlada con mayor facilidad, sin la generación de turbulencia excesiva o vibraciones en el equipo (Goyder, 2002). Los valores obtenidos mediante este modelo muestran congruencia con los calculados por Zhang et al. (2009), Taher et al. (2012) y Jian et al. (2015), para intercambiadores de helicoidales, mientras que para el caso del calentador de bafles verticales, Ozden et al. (2010) reportan valores simulares para intercambiadores de tubos y coraza con este tipo de bafles.

\section{CONCLUSIONES}

En el presente trabajo se realizó el cálculo del coeficiente de transferencia de calor para 4 configuraciones de calentadores con una resistencia interna basado en herramientas de dinámica de fluidos computacional. El cálculo del coeficiente de transferencia de calor mostró una dependencia lineal respecto al flujo volumétrico en la alimentación para las configuraciones con bafles tipo hélice y sin bafles. Para el caso de la configuración con bafles verticales los coeficientes de transferencia de calor muestran ser mayores debido al incremento en la turbulencia, mostrando que las condiciones hidrodinámicas y fenómenos de turbulencia son de gran impacto sobre los coeficientes de transferencia de calor. El uso de bafles helicoidales puede lograr resultados satisfactorios con menores caídas de presión debido a la reducción sustancial en los cambios de velocidad. Esta situación permite mantener una velocidad superficial mayor y más uniforme sobre en el fluido, sin caídas de presión excesivas. Esta investigación muestra la ventaja del uso de herramientas computacionales en la mejora que este tipo de sistemas eléctricos, pudiendo ser empleada para el análisis y optimización de calentadores industriales con un bajo costo de implementación.

\section{REFERENCIAS}

Alvis, A., I. Caicedo y P. Peña, Determinación del Coeficiente de Transferencia de Calor a Través de una Aplicación de Computadoras, doi: 10.1612/inf.tecnol.4367it.09, Inf. Tecnol. (en línea), 21(5), 13-20, (2010)

Andersson, B., R. Andersson, L. Håkansson, M. Mortensen, R. Sudiyo, y B. V. Wachem, Computational Fluid Dynamics for Engineers, 100-101, New York, Cambridge University Press, (2012)

Bhutta, M. M. A., N. Hayat, M. H. Bashir, A. R. Khan, K. N. Ahmad, y S. Khan, CFD applications in various heat exchangers design: A review, doi: 10.1016/j.applthermaleng.2011.09.001, Appl. Therm. Eng., 32, 1-12, (2012)

Bird, R. B., W. E Stewart, y E. N. Lightfoot, Transport Phenomena, 2ª edición, 423-437, John Wiley \& Sons, (2006).

COMSOL Inc, Chapter 6: Materials, COMSOL Multiphysics User's Guide, (2016)

Chen, Y.-P., Y.-J. Sheng, C. Dong, y J.-J. Wu, Numerical simulation on flow field in circumferential overlap trisection helical baffle heat exchanger, doi: 10.1016/j.applthermaleng.2012.07.031, Appl. Therm. Eng., 50, 1035-1043, (2013)

Dong, C., Y.-P. Chen y J.-F. Wu, Influence of baffle configurations on flow and heat transfer characteristics of trisection helical baffle heat exchangers, doi: 10.1016/j.enconman.2014.08.005, Energy Convers. Manage., 88, 251-258, (2014)

Dong, C., Y.-P. Chen y J.-F. Wu, Flow and heat transfer performances of helical baffle heat exchangers with different baffle configurations, doi: 10.1016/j.applthermaleng.2015.01.070, Appl. Therm. Eng., 80, 328-338, (2015)

EIA (U.S. Energy Information Administration), Annual Energy Outlook 2015 (AEO2015, en línea: http://www.eia.gov/oiaf/aeo, acceso 10 de enero 2015). Washington, DC, (2015)

Elecro, EVO Swimming Pool Heater (en la web: http://www.elecro.co.uk/in-line-swimming-pool-heater, acceso 10 de enero 2015), UK, (2016)

Friedler, F., Process integration, modelling and optimisation for energy saving and pollution reduction, doi: 10.1016/j.applthermaleng.2010.04.030, Appl. Therm. Eng., 30(16), 2270-2280, (2010)

Goyder, H. G. D., Flow-Induced Vibration in Heat Exchangers, doi: 10.1205/026387602753581971, Chem. Eng. Res. Des., 80(3), 226-232, (2002)

Ismail, L. S., y R. Velraj, Studies on Fanning Friction (f) and Colburn (j) Factors of Offset and Wavy Fins 
Compact Plate Fin Heat Exchanger-A CFD Approach, doi: 10.1080/10407780903507957, Numer. Heat Transfer, Part A, 56(12), 987-1005, (2009)

Jīn, Z. X., Anti-freezing and defreezing electric heating tube for solar water heater, CN2673022 Y, CN 200320107666, 19 de Enero (2005)

Kays, W. M. Turbulent Prandtl Number-Where Are We?, doi: 10.1115/1.2911398, J. Heat Transfer, 116(2), 284295, (1994)

Kim, D. E., M. H. Kim, J. E. Cha, y S. O. Kim, Numerical investigation on thermal-hydraulic performance of new printed circuit heat exchanger model, doi: 10.1016/j.nucengdes.2008.08.002, Nucl. Eng. Des., 238, 3269-3276, (2008)

Kim, M. I., Y. Lee, B. W. Kim, D. H. Lee y W. S. Song, CFD modeling of shell-and-tube heat exchanger header for uniform distribution among tubes, doi: 10.1007/s11814-009-0060-7, Korean J. Chem. Eng., 26(2), 359-363, (2009)

Kumar, V., S. Saini, M. Sharma y K. D. P. Nigam, Pressure drop and heat transfer study in tube-in-tube helical heat exchanger, doi: 10.1016/j.ces.2006.01.039, Chem. Eng. Sci., 61, 4403-4416, (2006)

Launder, B. E. y D. B. Spalding, The numerical computation of turbulent flows, doi: 10.1016/00457825(74)90029-2, Comput.Methods in Appl.Mech.Eng., 3(2), (1974)

Lei, Y.-G., Y.-L. He, P. Chu y R. Li, Design and optimization of heat exchangers with helical bafles, doi: 10.1016/j.ces.2008.05.044, Chem. Eng. Sci., 63, 4386-4395, (2008)

Lisboa, P. F., J. Fernandes, P. C. Simões, J. P. B. Mota y E. Saatdjian, Computational-fluid-dynamics study of a Kenics static mixer as a heat exchanger for supercritical carbon dioxide, doi: 10.1016/j.supflu.2010.08.005, The Journal of Supercritical Fluids, 55, 107-115, (2010)

Movassag, S. Z., F. N. Taher, K. Razmi, y R. T. Azar, Tube bundle replacement for segmental and helical shell and tube heat exchangers: Performance comparison and fouling investigation on the shell side, doi: 10.1016/j.applthermaleng.2012.10.025, Appl. Therm. Eng., 51, 1162-1169, (2013)

Nasr, M. R. J., y A. Shafeghat, Fluid flow analysis and extension of rapid design algorithm for helical baffle heat exchangers, doi: 10.1016/j.applthermaleng.2007.10.021, Appl. Therm. Eng., 28, 1324-1332, (2008)

Omer, A. M. Energy, environment and sustainable development, doi: 10.1016/j.rser.2007.05.001, Renew. Sust. Energ. Rev., 12(9), 2265-2300, (2008)

Ozden, E., e I. Tari, Shell side CFD analysis of a small shell-and-tube heat exchanger, doi: 10.1016/j.enconman.2009.12.003, Energy Convers. Manage., 51, 1004-1014, (2010)

Petra, C. G., O. Schenk, M. Lubin y K. Gäertner, An Augmented Incomplete Factorization Approach for Computing the Schur Complement in Stochastic Optimization, doi: 10.1137/130908737, SIAM J. Sci. Comput., 36(2), C139-C162, (2014)

Reichl, M. y A. Pleschinger, Dynamic flow heater, US20130202279 A1, US 13/634,191, 8 de agosto (2013).

Rodríguez-Toral, M. Á., y C. Heard, Aplicación de "bafles"1 helicoidales y tubos de bajo "aletado"2 al enfriamiento de gases. Tecnología, Ciencia, Educación (en línea), 27 (0186-6036), (2012)

Ryan, D., R. Long, D. Lauf, M. Ledbetter y A. Reeves, ENERGY STAR Water Heater Market Profile, Silver Spring, MD: D\&R International, (2010)

Shi, Y.-L., J.-J. Ji y C.-L Zhang, Semiporous Media Approach for Numerical Simulation of Flow through LargeScale Sparse Tubular Heat Exchangers, doi: 10.1080/10789669.2010.10390924, HVAC\&R Research, 16(5), 617-628, (2010)

$\mathrm{Su}, \mathrm{H}$., Large power carbon fiber electric heating tube for industrial use, CN101389163 B, CN 200810158363 , 02 de noviembre (2011)

Taher, F. N., S. Z. Movassag, K. Razmi y R. T. Azar, Baffle space impact on the performance of helical baffle shell and tube heat exchangers, doi: 10.1016/j.applthermaleng.2012.03.042. Appl. Therm. Eng., 44, 143-149. (2012). 
Wang, S., J. Wen, H. Yang, Y. Xue y H. Tuo, Experimental investigation on heat transfer enhancement of a heat exchanger with helical baffles through blockage of triangle leakage zones, doi: 10.1016/j.applthermaleng2014.03.017, Appl. Therm. Eng., 67, 122-130, (2014)

Wang, Y., Z. Liu, S. Huang, W. Liu y W. Li, Experimental investigation of shell-and-tube heat exchanger with a new type of bafles, doi: 10.1007/s00231-010-0590-x, Heat Mass Transfer., 47(7), 833-839, (2011)

Weaver, D. S., y J. A. Fitzpatrick, A review of cross-flow induced vibrations in heat exchanger tube arrays, doi: 10.1016/S0889-9746(88)90137-5, J. Fluid. Struct., 2(1), 73-93, (1988)

Wasewar, K. L., S. Hargunani, P. Atluri y N. Kumar, CFD Simulation of Flow Distribution in the Header of PlateFin Heat Exchangers, doi: 10.1002/ceat.200700180, Chem. Eng. Technol., 30(10), 1340-1346, (2007)

Wen, J., y Y. Li, Study of flow distribution and its improvement on the header of plate-fin heat exchanger, doi: 10.1016/j.cryogenics.2004.04.009, Cryogenics, 44, 823-831, (2004)

Wen, J., H. Yang, S. Wang, Y. Xue y X. Tong, Experimental investigation on performance comparison for shelland-tube heat exchangers with different bafles, doi: 10.1016/j.ijheatmasstransfer.2014.12.071, Int. J. Heat Mass Transfer, 84, 990-997, (2015)

Xiao, X., L. Zhang, X. Li, B. Jiang, X. Yang y Y. Xia, Numerical investigation of helical baffles heat exchanger with different Prandtl number fluids, doi: 10.1016/j.ijheatmasstransfer.2013.04.001, Int. J. Heat Mass Transfer, 63, 434-444, (2013)

Yang, J.-F., M. Zeng y Q.-W Wang, Effects of sealing strips on shell-side flow and heat transfer performance of a heat exchanger with helical bafles, doi: 10.1016/j.applthermaleng.2013.11.064, Appl. Therm. Eng., 64, 117-128, (2014)

Yang, J., L. Ma, J. Bock, A. M. Jacobi, y W. Liu, A comparison of four numerical modeling approaches for enhanced shell-and-tube heat exchangers with experimental validation, doi: 10.1016/j.applthermaleng.2014.01.035, Appl. Therm. Eng., 65, 369-383, (2014)

You, Y., A. Fan, S. Huang, y Liu, W. (2012). Numerical modeling and experimental validation of heat transfer and flow resistance on the shell side of a shell-and-tube heat exchanger with flower baffles. Int. J. Heat Mass Transfer, 55, 7561-7569. doi: 10.1016/j.ijheatmasstransfer.2012.07.058

Zhang, J.-F., S.-L. Guo, Z.-Z. Li, J.-P. Wang, Y.-L. He, y W.-Q. Tao, Experimental performance comparison of shell-and-tube oil coolers with overlapped helical baffles and segmental bafles, doi: 10.1016/j.applthermaleng.2013.04.009, Appl. Therm. Eng., 58(1-2), 336-343, (2013)

Zhang, J.-F., Y.-L. He y W.-Q. Tao, 3D numerical simulation on shell-and-tube heat exchangers with middleoverlapped helical baffles and continuous baffles - Part I: Numerical model and results of whole heat exchanger with middle-overlapped helical bafles, doi: 10.1016/j.jjheatmasstransfer.2009.07.006, Int. J. Heat Mass Transfer, 52, 5371-5380, (2009)

Zhang, J.-F., B. Li, W.-J. Huang, Y.-G. Lei, Y.-L. He y W.-Q. Tao, Experimental performance comparison of shell-side heat transfer for shell-and-tube heat exchangers with middle-overlapped helical baffles and segmental bafles, doi: 10.1016/j.ces.2008.12.018, Chem. Eng. Sci., 64(8), 1643-1653, (2009)

Zhang, Z., y Y. Li, CFD simulation on inlet configuration of plate-fin heat exchangers, doi: 10.1016/S00112275(03)00179-6, Cryogenics, 63, 673-678, (2003) 